\title{
Suppressing Vortex Induced Vibrations of Wind Turbine Blades with Flaps
}

\author{
Horcas, Sergio González; Madsen, Mads; Sørensen, Niels N.; Zahle, Frederik
}

Published in:

Recent Advances in CFD for Wind and Tidal Offshore Turbines

Link to article, DOI:

10.1007/978-3-030-11887-7_2

Publication date:

2019

Document Version

Peer reviewed version

Link back to DTU Orbit

Citation (APA):

Horcas, S. G., Madsen, M., Sørensen, N. N., \& Zahle, F. (2019). Suppressing Vortex Induced Vibrations of Wind Turbine Blades with Flaps. In E. Ferrer, \& A. Montlaur (Eds.), Recent Advances in CFD for Wind and Tidal Offshore Turbines (pp. 11-24). Springer. Springer Tracts in Mechanical Engineering (STME) https://doi.org/10.1007/978-3-030-11887-7_2

\section{General rights}

Copyright and moral rights for the publications made accessible in the public portal are retained by the authors and/or other copyright owners and it is a condition of accessing publications that users recognise and abide by the legal requirements associated with these rights.

- Users may download and print one copy of any publication from the public portal for the purpose of private study or research.

- You may not further distribute the material or use it for any profit-making activity or commercial gain

- You may freely distribute the URL identifying the publication in the public portal 
6th European Conference on Computational Mechanics (ECCM 6) 7th European Conference on Computational Fluid Dynamics (ECFD 7)

1115 June 2018, Glasgow, UK

\title{
SUPPRESSING VORTEX INDUCED VIBRATIONS OF WIND TURBINE BLADES WITH FLAPS
}

\section{S.G. HORCAS, M.H.A. MADSEN, N.N. SØRENSEN and F. ZAHLE}

DTU Wind Energy, Frederiksborgvej 399, 4000 Roskilde, sgho@dtu.dk, www.dtu.dk

Key words: AVATAR, Computational Fluid Dynamics, Flaps, Fluid Structure Interaction, Vortex Induced Vibrations, Wind Energy

\begin{abstract}
The present paper describes an exploratory work aiming to analyze the impact of trailing edge flaps activation on Vortex Induced Vibrations (VIV) suppression. A computational study of the VIV of the AVATAR rotor blade, a 10MW design suitable for offshore locations, was performed. A Fluid Structure Interaction (FSI) approach was adopted for the simulations, coupling an Improved Delayed Detached Eddy Simulations (IDDES) flow solver with a beambased structural model. Initial simulations based on the clean geometry identified significant edgewise VIV for certain free stream velocity and flow inclination angles. The inflow conditions showing the maximum amplitude of blade vibrations were used in order to test several trailing edge flap geometries and operating angles. The best flap configuration found in that parametric study managed to suppress the VIV phenomenon. However, when assessing a wider range of inflow conditions, the amplitudes of vibration of the blade equipped with flaps were found to be equivalent to the ones obtained for its clean counterpart. It is therefore concluded that a re-calibration of the flap operating angle should be required in order to adapt it to the considered wind speed and wind direction.
\end{abstract}

\section{INTRODUCTION}

Due to the flexibility of their blades, it is presumed that modern horizontal wind turbines may be susceptible to Vortex Induced Vibrations (VIV), a phenomenon which could ultimately lead to structural failure of the blade. This statement was supported by the recent high-fidelity simulations of Heinz et al. (2016b), where the authors analyzed the DTU 10MW reference wind turbine blade under realistic inflow conditions. The present paper constitutes a first exploratory work in order to use flow control devices for the suppression of this phenomenon. In particular, the influence of the installation of trailing edge flaps is assessed. Previous studies based on Computational Fluid Dynamics (CFD) methods have shown the potential of these devices for load alleviation during the operation of multi-megawatt wind turbines Jost et al. (2016); Barlas et al. (2016)]. However, to the best of authors' knowledge no publication regarding the use of trailing edge flaps for VIV suppression can be found in the literature.

In order to assess the performance of trailing edge flaps with regards to VIV suppression, a comprehensive numerical study was performed. It was based on the AVATAR reference rotor Lekou et al. (2015)], that is equipped with a $100 \mathrm{~m}$ blade. The chosen computational method, presented in Section 2, relied on the coupling of a fluid and a structure solvers by means of a Fluid Structure Interaction (FSI) approach. Three different sets of FSI simulations were performed. 
First computations, detailed in Section 3, were based on the clean blade geometry. The aim for said simulations was to identify the particular inflow conditions which lead to the maximum amplification of vibrations, thereby highlighting the mechanisms involved in this phenomenon. A subsequent set of simulations accounting for trailing edge flaps was carried out. Due to time constraints, the deflected flaps geometry was estimated based on a simple analytical expression. The results of these simulations are presented in Section 4, and comprise both an assessment of the performance of the flap for VIV suppression as well as a quantification of the influence of the geometric parameters characterizing this device. For these simulations, the inflow conditions exhibiting the maximum amplification for the clean geometry were adopted. Finally, Section 5 compiles a wider assessment of the flaps installation with regards to VIV suppression. The initially studied analytical representation of the flaps was replaced by the use of a Free Form Deformation (FFD) method. This technology, introduced in the fluid solver in the framework of the present work, establishes a much more versatile approach for future applications. An initial comparison with the performance of the analytic flap version was performed, followed by the assessment of the inflow conditions range considered for the clean geometry.

\section{COMPUTATIONAL SET-UP}

The fluid was modeled with the CFD solver EllipSys3D [Michelsen (1992, 1994); Sørensen (1995)], which is a finite volume code that solves the Navier Stokes equations on a structured grid and in curvilinear coordinates. In all the simulations presented in this work, a zonal Improved Delayed Detached Eddy Simulation (IDDES) was employed in order to deal with turbulence Menter and Kuntz (2004)]. In this way, a Reynolds-Averaged Navier Stokes (RANS) model was employed close to the blade surface and a Large Eddy Simulation (LES) approach was followed far away from the boundary layer (where the turbulent length scales become larger than the used grid resolution). For the region involving the RANS method, the k- $\omega$ SST turbulence model of Menter (1994) was used.

A three level grid sequence was used during the simulation to speed up the development of the wake flow. A time step of $1.5 \cdot 10^{-3} s$ was employed, with a total simulation time of $68 s$. The air density was fixed to $1.225 \mathrm{~kg} \mathrm{~m}^{-3}$, and the dynamic viscosity was set to $1.7879 \cdot 10^{-5}$ $\mathrm{kg} \mathrm{m}^{-1} \mathrm{~s}^{-1}$. An inlet/outlet strategy was used for the boundary conditions. For the former region, a value of $1 \cdot 10^{5} \mathrm{~s}^{-1}$ was imposed for the specific dissipation $\omega$, while the turbulent kinetic energy was set to $1 \cdot 10^{-2} \mathrm{~m}^{2} \mathrm{~s}^{-2}$.

The same structured CFD mesh was used for all the simulations. It accounted for 240 blocks of $32 \times 32 \times 32$ cells, leading to a total of 7.8 millions for the complete mesh. The chordwise direction was discretized with 256 cells (16 of them lying on the trailing edge). A boundary layer clustering was taken into account, with an imposed first cell height on the order of $10^{-6}$ $m$. This allowed to tackle y+ values lower than 1 for the considered simulation conditions. The surface mesh is depicted in Figure 1, together with a detail of a section cut at half span.

The CFD model was coupled with a structural model of the blade by means of the staggered FSI approach presented in Heinz et al. (2016b). The inner CFD mesh deformation process was enhanced in the framework of the present work. A generalized analytical approach was implemented, where the deformation vector is smoothed based on a hyperbolic tangent function. The blade structure was handled by the commercial solver HAWC2 [Larsen and Hansen (2015)], and relied on a series of Timoshenko beam elements. The effect of both cone and tilt angles were neglected in the present study. 


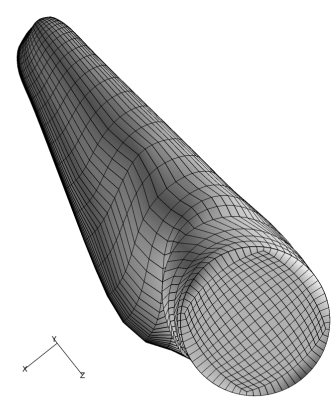

(a) Surface mesh

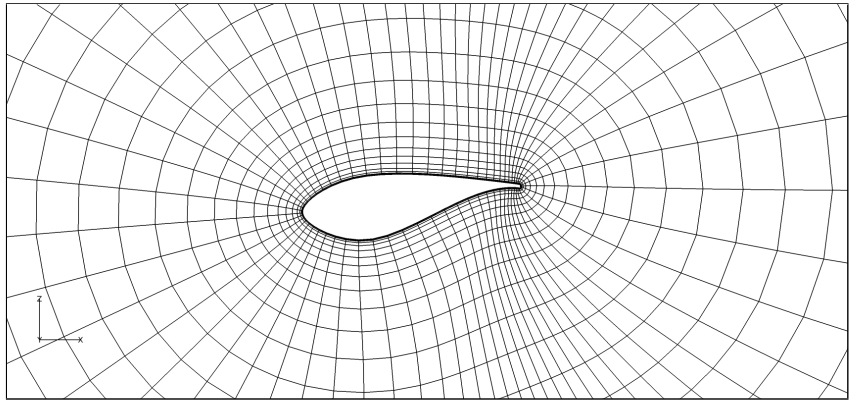

(b) Half span cut

Figure 1: Overview of CFD mesh. For clarity purposes, only 1 out of 4 grid lines are displayed.

All the presented FSI computations were run on the Jess high-performance computing cluster owned by DTU. A total of 240 processors running at $2.8 \mathrm{GHz}$ were used per computation, requiring a wall clock time of approximately 15 hours.

\section{RESULTS FOR THE CLEAN GEOMETRY}

Previous computational studies of large wind turbines revealed that the main set-up parameters influencing the dynamic behavior of a standstill blade are related to the inflow conditions Heinz et al. (2016ba)]. For both aforementioned studies, maximum vibrations amplifications were observed for an angle of attack at the blade tip slightly higher than $90 \mathrm{deg}$. Hence, it was decided to fix this angle, referred to in this work as pitch angle $\theta$, to $95 \mathrm{deg}$. Together with the pitch angle, both the absolute value of freestream velocity $\left\|U_{\infty}\right\|$ and its inclination angle with respect to the blade axis $\psi$ were identified as the main actors triggering the VIV phenomenon. A graphical representation of these parameters is depicted in Figure 2. All the simulations included in this work are labeled as $U X X_{-} I Z Z$, where $X X$ refers to the value of $\left\|U_{\infty}\right\|$ and $Z Z$ to $\psi$. In order to analyze the behavior of the clean blade geometry with regards to VIV, two consecutive parametric studies were performed.

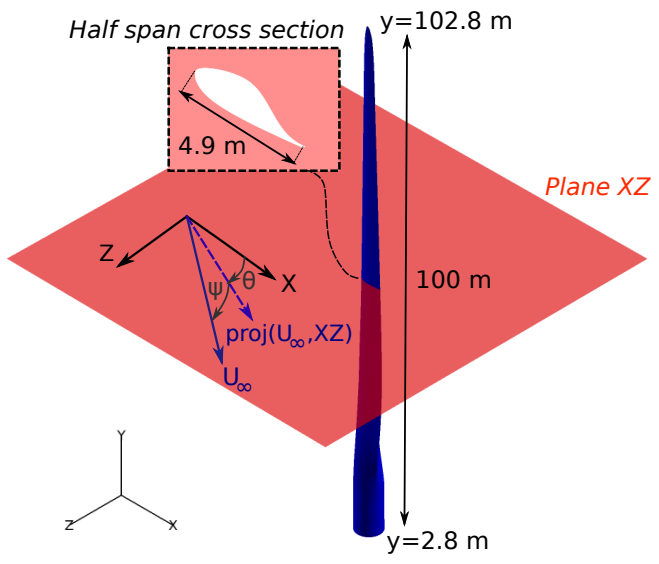

Figure 2: Description of considered simulation parameters for the different test cases.

The first parametric study concerned the effect of the inclination angle $\psi$. In particular, angles ranging from 0 to $80 \mathrm{deg}$ were considered. A common free stream velocity $\left\|U_{\infty}\right\|$ was 
used for all the computations. It was estimated based on an analytical computation, aiming at the generation of outboard shedding frequencies at the vicinity of the first edgewise mode of the blade (i.e. $0.91 \mathrm{~Hz}$ ), where maximum VIV are expected. Indeed, the natural frequency of shedding $f_{\text {shnat }}$ of an inclined flow with an angle $\psi$ can be related to the Strouhal number $S t$ by means of the independence principle, which is valid for small angles [Hoang et al. (2015)], as:

$$
f_{\text {shnat }}=\frac{\left\|U_{\infty}\right\| \cos (\psi) S t}{c}
$$

where $c$ refers to a reference length (in this case the chord). Assuming a Strouhal number of approximately 0.16 [Heinz et al. (2016ba)], taking as reference chord the value at $85 \%$ of blade span (i.e. $2.96 \mathrm{~m}$ ) and assuming a complete synchronization of the shedding frequency and the first edgewise mode for an inclination angle of $40 \mathrm{deg}$, a $\left\|U_{\infty}\right\|$ of $22 \mathrm{~m} \mathrm{~s}^{-1}$ was selected for this initial study.

The second parametric study concerned the variation of the free stream velocity $\left\|U_{\infty}\right\|$. In particular, values ranging from 10 to $40 \mathrm{~m} \mathrm{~s}^{-1}$ were considered. The value of the inclination angle $\psi$ was fixed to $40 \mathrm{deg}$, consistently with the assumptions made in the choice of the free stream velocity of the first parametric study.

Figure 3 depicts, for both parametric studies, the maximum observed peak-to-peak values of the blade tip deflection transients. Since the VIV phenomenon is expected for the edgewise motion, only the tangential component with respect to the rotor plane is shown (corresponding in this case to the $\mathrm{x}$ direction). Regarding the parametric study of the inclination angle (Figure 3a), the results reveal a significant amplification of the edgewise deflection for the U22_I40 simulation, with a maximum peak-to-peak value of $5.55 \mathrm{~m}$ (corresponding to 9.25 times the blade tip chord). The variation of the inclination angle led to a decrease of the computed deflections, with negligible amplitudes for both 0 and $80 \mathrm{deg}$. An analogous observation can be made for the parametric study concerning the influence of the free stream velocity, shown in Figure $3 \mathrm{~b}$,

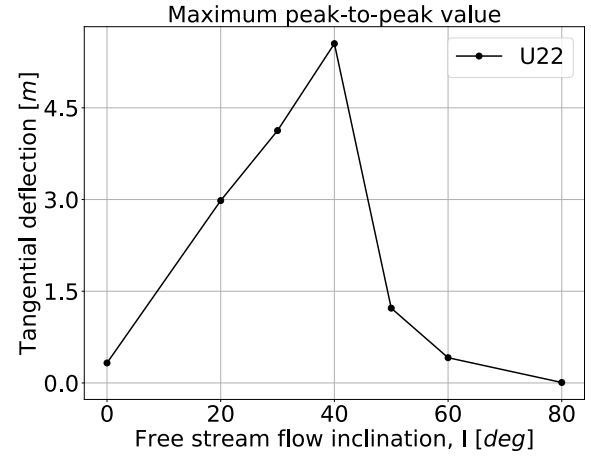

(a) Effect of inclination angle

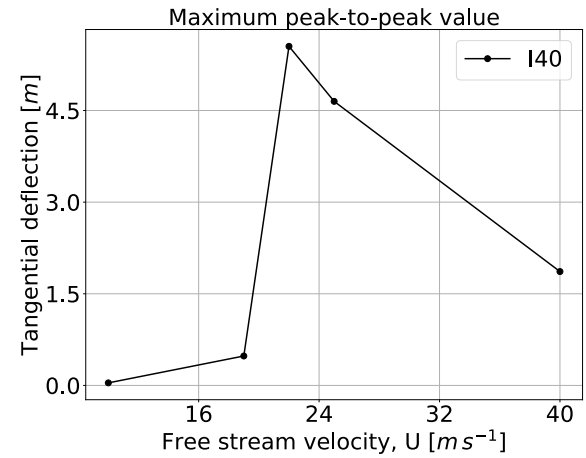

(b) Effect of free stream velocity

Figure 3: Maximum peak-to-peak value of the tangential blade tip deflection transients. Clean blade.

In Figure 4, the Power Spectral Density (PSD) of the blade sectional loading time series is shown for several simulations. Due to its potential impact on edgewise vibrations, only the $\mathrm{x}$ component of the load was considered. Additionally, the plots were limited to a high span range. Results are displayed by means of color plots, with the ordinates representing the blade radial position and the corresponding load frequencies included in the abcissae. All the sectional PSDs 
were computed by means of the Welch method [Welch $(1967)]$, and the results were linearly scaled (so that the peaks height become an estimate of the RMS amplitude). For the U22_I40 simulation

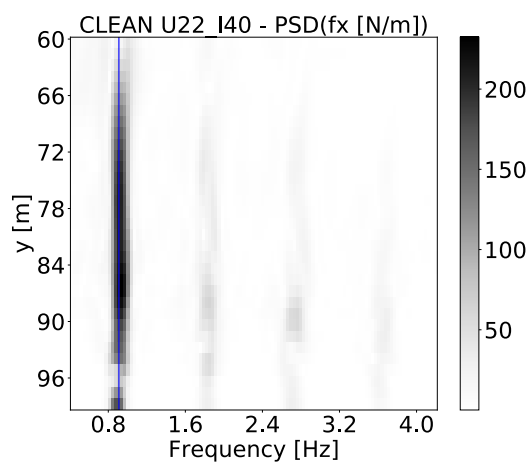

(a) U22_I40

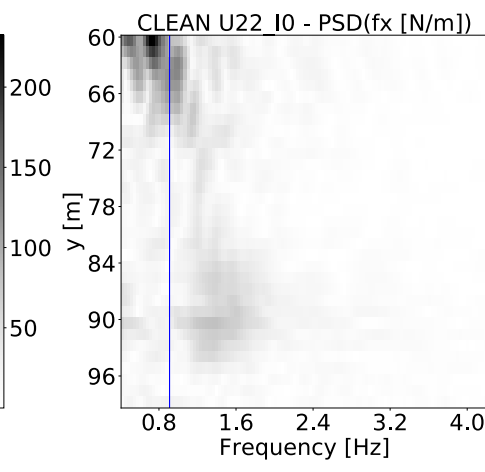

(b) U22_I0

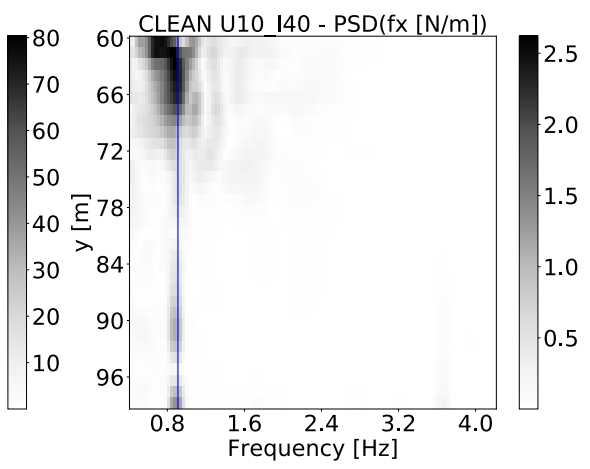

(c) U10_I40

Figure 4: PSDs of the $\mathrm{x}$ component of blade sectional loading. Clean blade. A vertical line corresponding to the first edgewise mode of the blade was added for reference.

(Figure 4a), that exhibited the highest vibration amplitudes, important loads fluctuations were observed. Additionally, a significant correlation of the loading frequencies along the outboard region of the blade was found, corresponding to the first edgewise mode of the blade. This fact, already observed in Heinz et al. (2016b) and Heinz et al. (2016a), is assumed to be related to the the existence of the spanwise flow. Indeed, the maximum fluctuations were obtained at $85 \%$ of the blade span, as initially predicted by Equation 1, corresponding to $\mathrm{y}=87.8 \mathrm{~m}$. This significant fluctuation was then convected all along the span, resulting in a high frequency content around the first edgewise mode for the outboard region of the blade. Much lower PSD levels were obtained for the U22_I0 simulation (Figure 4b), were the absence of a spanwise flow component led to the decorrelation of the loading frequencies. Finally, the results of the U10_40 simulation are depicted in Figure 4c. For this simulation, a considerable difference between the natural shedding frequencies of the flow and the first edgewise mode is expected, due to the low value of the free stream velocity. This could explain the low PSD values computed for this simulation. Indeed, a suppression of the Von Karman street for the outboard region of the blade was observed. This is illustrated in Figure 5 by means of the iso-surfaces of the absolute value of vorticity, where analogous results for the two other simulations are included for reference.

\section{PRELIMINARY STUDY BASED ON AN ANALYTIC FLAP}

In order to assess the performance of the introduction of flaps on the suppression of VIV, a preliminary study based on an analytical hinged flap definition was performed. Only the inflow conditions showing the maximum vibration amplitudes for the clean configuration, i.e. U22 and I40, were considered in this study. The flap deflection was introduced by means of a local deformation of the CFD mesh of the blade surface.

Two main geometrical parameters were considered: the flap length with respect to the chord and the spanwise extent with respect to the blade radius. In particular, values of $20 \%$ and $30 \%$ were considered for the former variable, labeled in this work as XC20 and XC30. Regarding the spanwise extent, values of $20 \%$ and $25 \%$ were considered, referred to in this work as $L 020$ and L025. For both cases, the flap was assumed to be centered at $75 \%$ of the blade radius. This led 


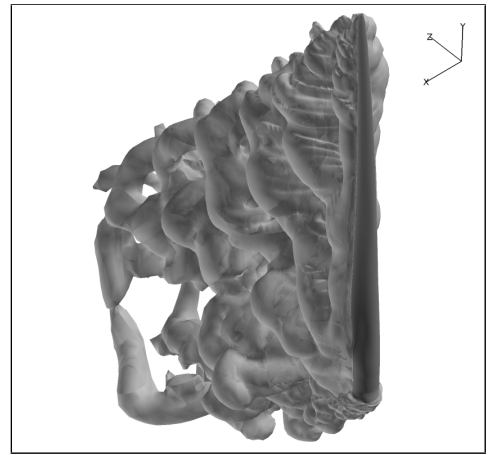

(a) U22_I40

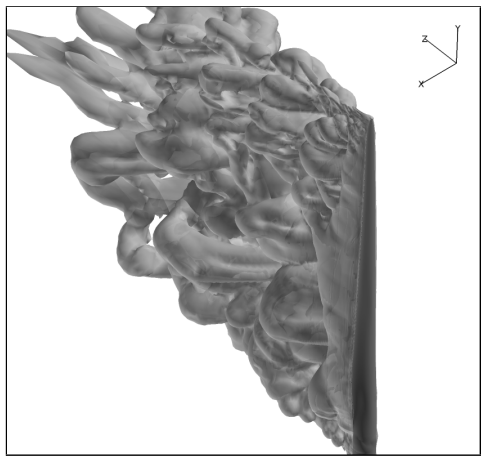

(b) U22_I0

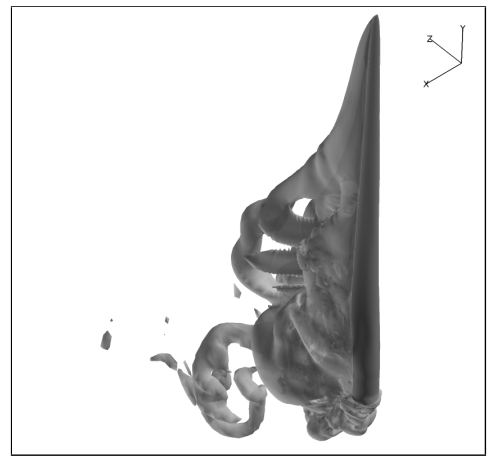

(c) U10_I40

Figure 5: Iso-surface of absolute value of vorticity at $2 s^{-1}$. Different inflow conditions for clean blade.

to a total radial extent ranging from $\mathrm{y}=68 \mathrm{~m}$ to $\mathrm{y}=88 \mathrm{~m}$ for the $L 020$ configuration, and from $\mathrm{y}=65 \mathrm{~m}$ to $\mathrm{y}=90 \mathrm{~m}$ for the $L 025$ case. Four different operating angles were also considered: -30 , -10, 10 and $30 \mathrm{deg}$. Those are labelled in this work as DN30, DN10, D10 and D30 respectively.

The deflected flap geometry was achieved by a linear interpolation of the clean mesh and one rotated according to the flapping angle (see Figure 6a). A fifth order polynomial smoothing function was applied from the position of the hinge point, estimated based on the value of the flap length, up to the trailing edge. This ensured a smooth transition of the suction and the pressure side curvatures, even if a higher effective flapping angle was expected. An analogous smoothing function was applied in the spanwise direction in order to ensure a smooth transition between the flapped and clean configurations (see Figure 6b).

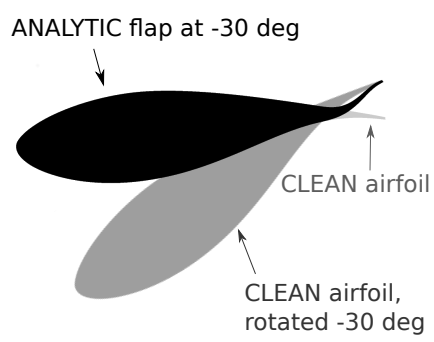

(a) Half of flap extent

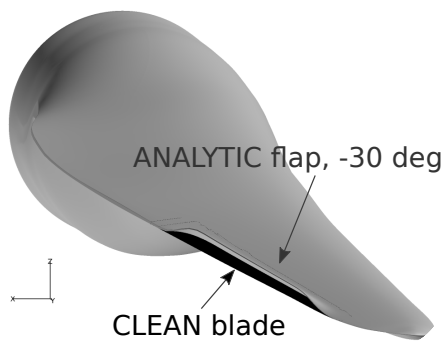

(b) $3 \mathrm{D}$ view

Figure 6: Geometry of the analytic flap XC20_L20 operating at -30 deg.

Figure 7 compiles the blade tip tangential deflection transients for the studied flapping geometries, superposed to the corresponding results for the clean configuration. It can be observed that, regardless the flap geometry, all the configurations operating at -30 deg managed to suppress the amplification of the blade vibration. The same remark can be made for the simulations accounting for a flap extent of $25 \%$ in the spanwise direction and operating at $10 \mathrm{deg}$. For both cases, the mechanism preventing the amplification of the blade vibration was assumed to be related to the suppression of the Von Karman street at the outboard region. This is illustrated in Figure 8 by means of the iso-surfaces of vorticity of a flap configuration undergoing VIV (XC20_L20_D30), and two set-up where the vibrations were suppressed (XC20_L20_DN30 and 


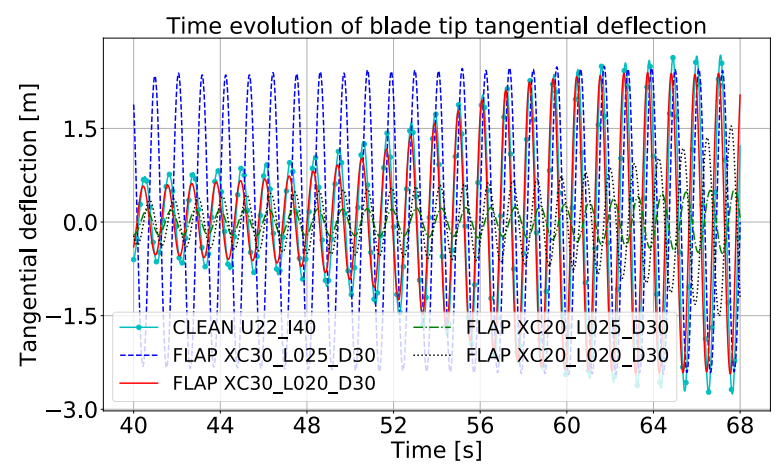

(a) Flap angle $30 \mathrm{deg}$

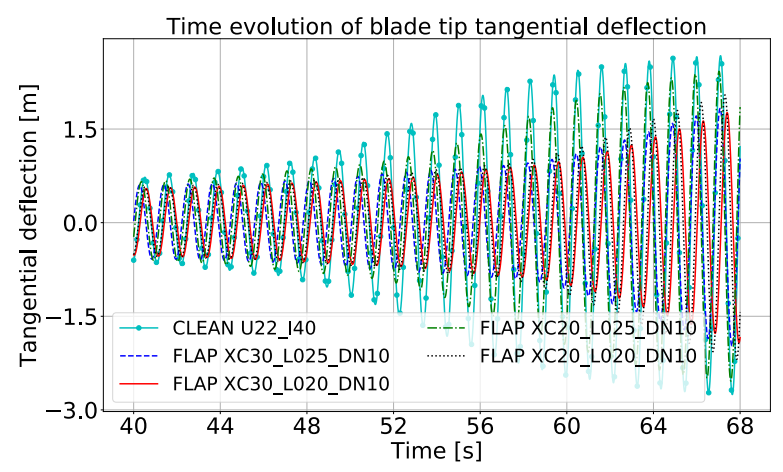

(c) Flap angle $-10 \mathrm{deg}$

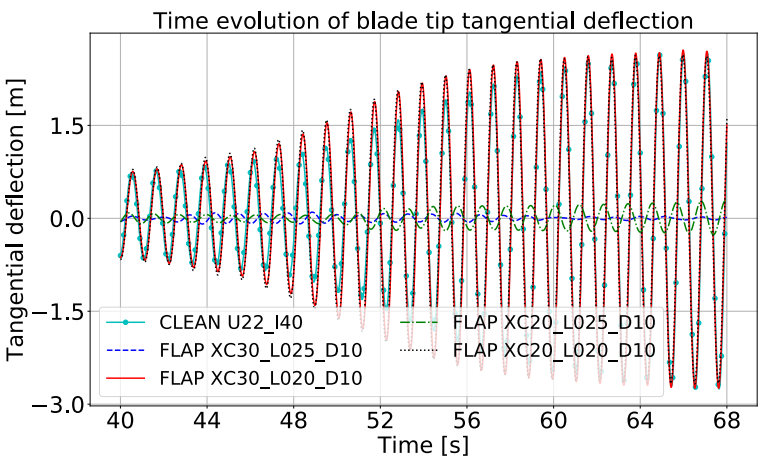

(b) Flap angle $10 \mathrm{deg}$

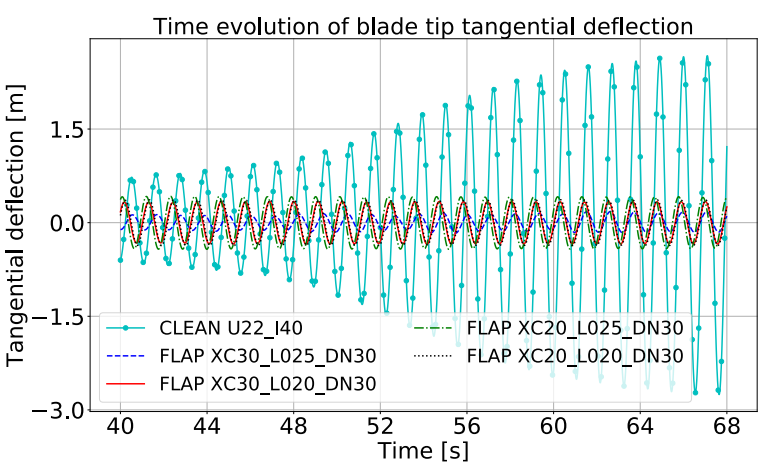

(d) Flap angle $-30 \mathrm{deg}$

Figure 7: Blade tip tangential deflection transient for different analytic flap configurations and operating angles.

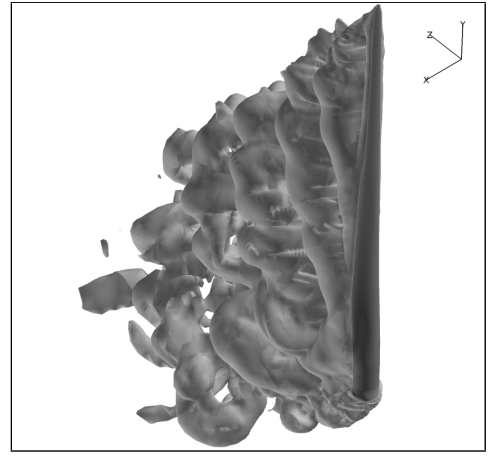

(a) XC20_L20_D30

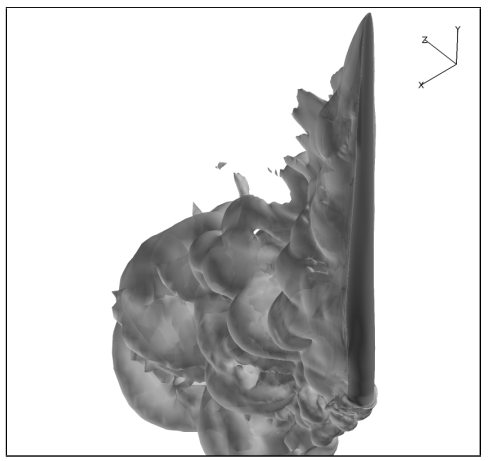

(b) XC20_L20_DN30

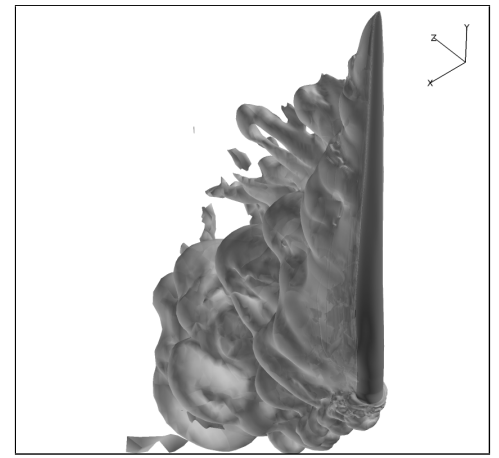

(c) XC20_L25_D10

Figure 8: Vorticity iso-surface at $2 s^{-1}$, blade equipped with different analytic flap geometries.

XC20_L25_D10). A more detailed visualization of the Von Karman street suppression is depicted in Figure 9, where the y component of the vorticity is shown for two different blade sections. For XC20_L20_DN30 and XC20_L25_D10 computations, the spanwise vortex generated at the blade tip remained attached up to approximately $65 \%$ of the blade span. For lower sections, not shown in this document, a Von Karman street similar to the one found for XC20_L20_D30 was developed. The detailed mechanism preventing the outboard region Von Karman street would 
require additional work, including a pure CFD study of a stiff blade configuration. However, the change of the Strouhal number induced by the flap geometry could be argued as a preliminary explanation. This is consistent with the reduction of VIV observed for the clean configuration at low wind speeds (see Section 3), where significant differences between the natural shedding frequencies of the blade and the first edgewise mode were expected. To the best of authors' knowledge, this is the first time that this complex three-dimensional flow behaviour is identified in the context of wind energy. However, an extensive literature dealing with inclined cylinders can be found. The existence of the spanwise vortex was identified, among others, in the experimental work of Matsumoto et al. (2001). The mitigation of VIV by the suppression of the Von Karman Street was reported in the DES simulations of Yeo and Jones (2010). This was achieved by equipping an inclined cylinder with strakes. Similar conclusions were found in the subsequent computational studies of Gioria et al. (2011) and Zhou et al. (2011).

The suppression of the Von Karman street had as a result a mitigation of the load fluctuations, explaining the low level of observed blade vibrations. This is illustrated in the PSD of the blade sectional loading of Figure 10. A common scale was used to facilitate the comparison.

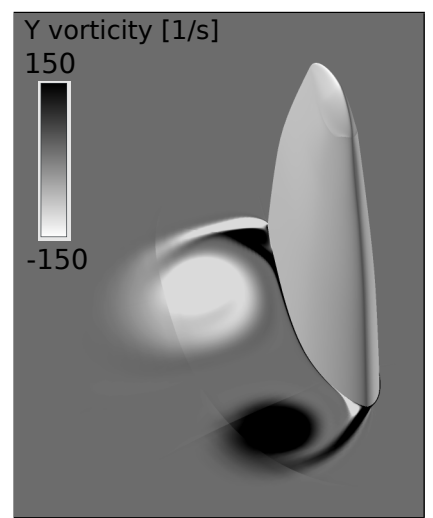

(a) XC20_L20_D30

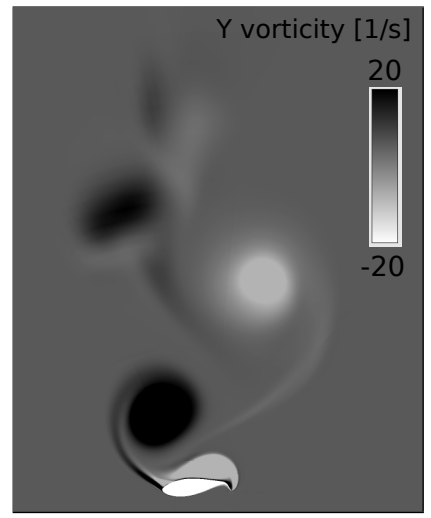

(d) XC20_L20_D30

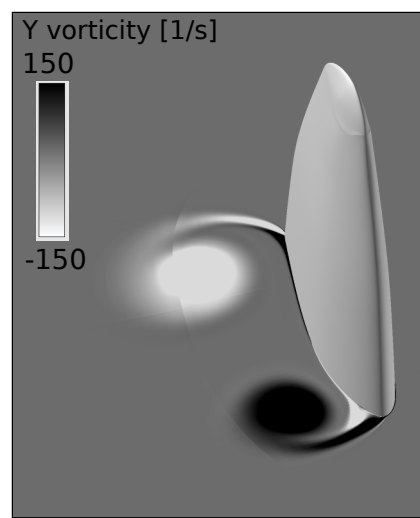

(b) XC20_L20_DN30

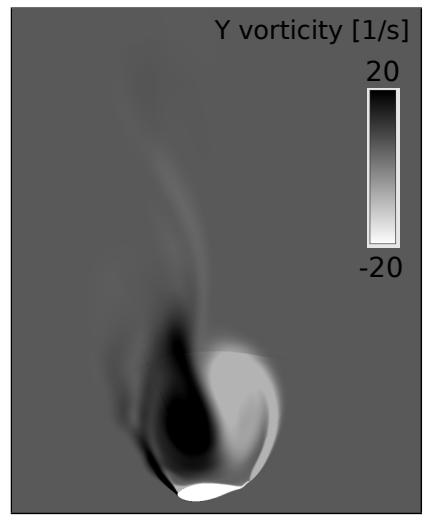

(e) XC20_L20_DN30

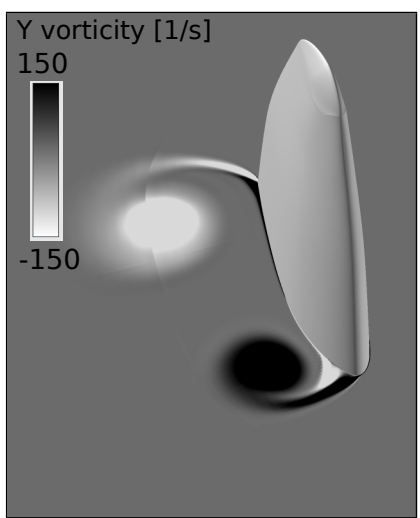

(c) XC20_L25_D10

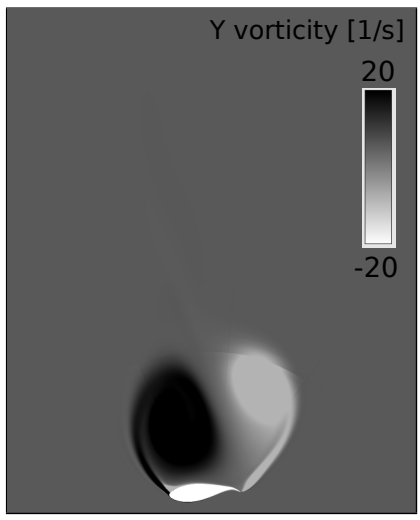

(f) XC20_L25_D10

Figure 9: $\mathrm{Y}$ vorticity component for section cuts located near the blade tip (upper row) and at $\mathrm{y}=77.9 \mathrm{~m}$ (lower row). Blade equipped with different analytic flap geometries. 


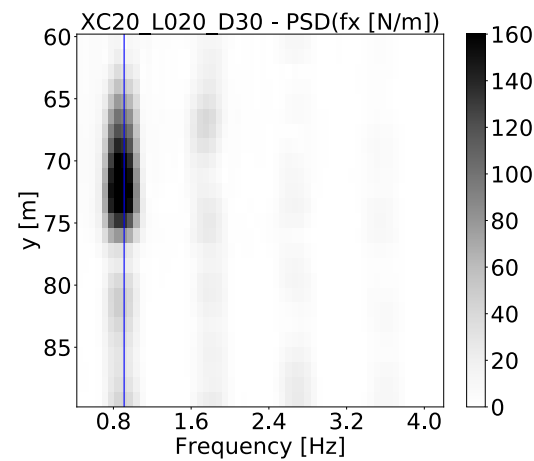

(a) XC20_L20_D30

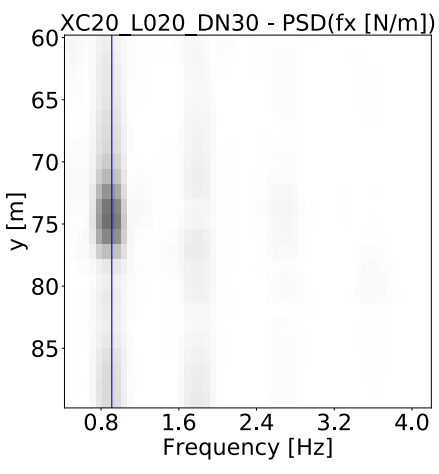

(b) XC20_L20_DN30

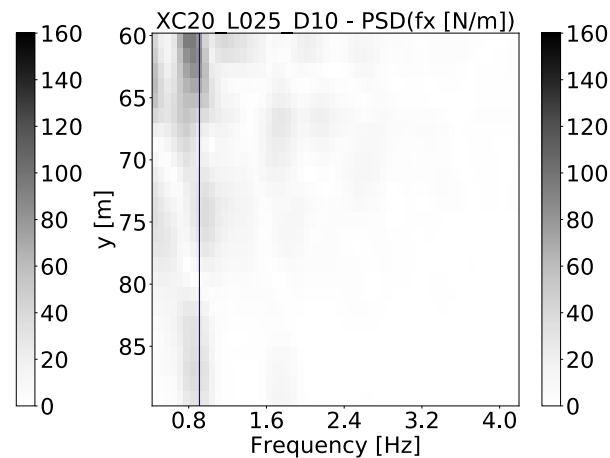

(c) XC20_L25_D10

Figure 10: PSDs of the $\mathrm{x}$ component of blade sectional loading. Includes several analytic flap configurations. A vertical line corresponding to the first edgewise mode of the blade was added for reference.

\section{INTRODUCTION OF THE FREE FORM DEFORMATION FLAP}

The preliminary study based on an analytical definition of the flap revealed a good potential for all the studied geometries when working at -30 deg. The XC20_L20 was therefore selected, due to its reduced dimensions, to be re-evaluated by means of the Free Deformation Deformation (FFD) method. This technique, originating from the field of computer graphics Sederberg and Parry (1986)], is a very lenient and versatile way of manipulating a given geometry. The most straight forward way of understanding the deformation mechanism is to think of the object as being wrapped in soft rubber. When one moves the rubber material (by moving given control points) the deformation is propagated inwards to deform all material inside the control box depending on the distance to the moved control points. There are many benefits of FFD including possible continuity control, volume preservation and analytically defined deformation gradients. The FFD utilities used in the present work are all from the FFDlib-toolbox which is developed at DTU Wind Energy to facilitate High-Fidelity Shape Optimization as well as general surface mesh deformation. In Figure 11a a box from FFDlib is shown along the trailing edge of the blade, which is displayed in black. By moving only a selection of control points (shown in white), a smooth transition from flapping region to original blade is obtained. Figure 11 shows a comparison of the analytic and FFD flap versions. It should be remarked that the FFD implementation accounted for a faster transition towards the rotated flap geometry. This results

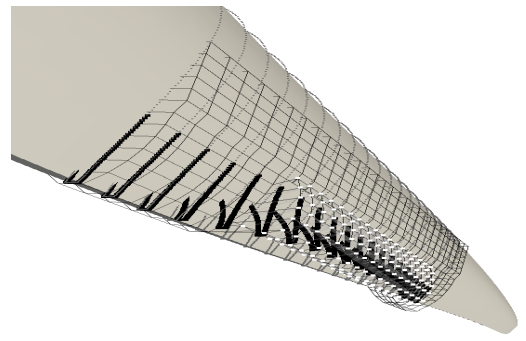

(a) FFD box

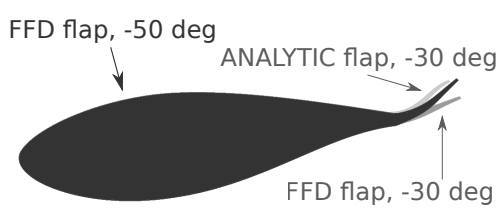

(b) Half of flap extent

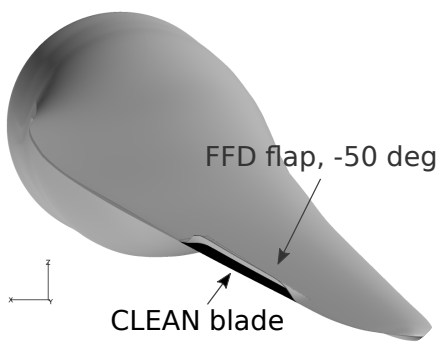

(c) $3 \mathrm{D}$ view

Figure 11: Geometry of the FFD flap XC20_L20 operating at -50 deg. 
in a lower (and more realistic) flapping angle. In particular, an analytic flap working at -30 deg seemed to correspond to the FFD counterpart operating at -50 deg.

First simulations were devoted to verify this observation under the U22_I40 inflow conditions. Figure 12 shows a comparison of the FFD flap working at several negative flapping angles. As expected, a good agreement was found for the FFD flap working at $-50 \mathrm{deg}$. This operation angle was kept for the final evaluation of the flap, were the whole set of inflow conditions presented in Section 3 was considered.

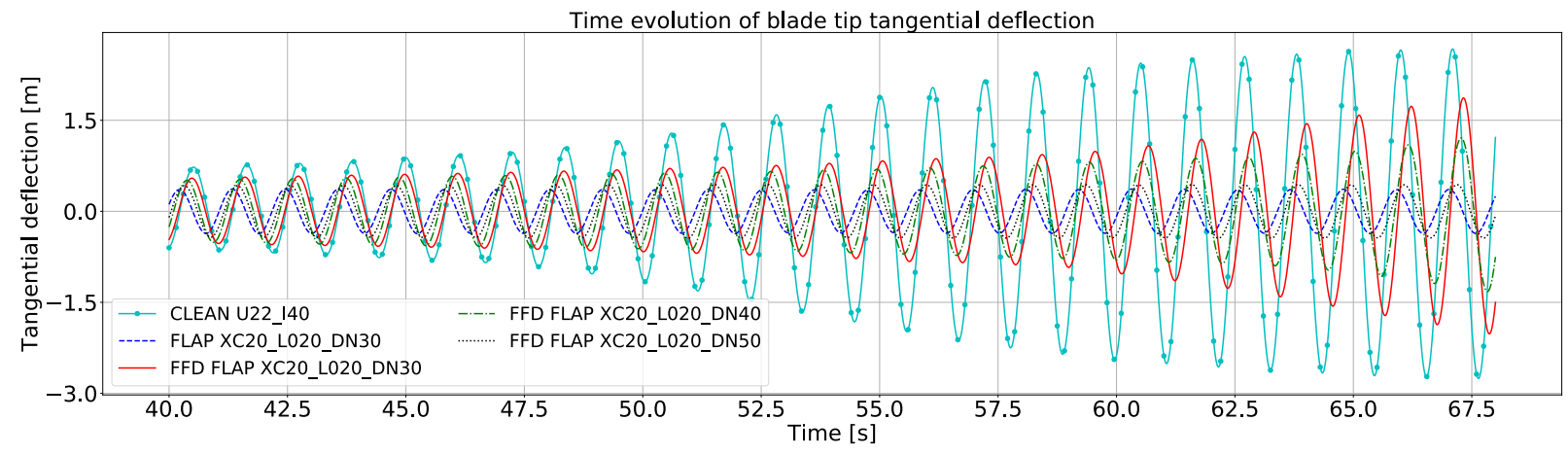

Figure 12: Blade tip tangential deflection transient for the analytic and FFD flaps. U22_I40 simulation.

Figure 12 depicts the maximum peak-to-peak values computed for the blade equipped with the FFD flap, compared to the clean geometry results. Outside the inflow conditions showing the maximum blade vibrations amplification, the behavior of the flapped blade was found to be equivalent to its clean counterpart. This reveals that the choice of the flapping angle performed in Section 4 should be re-assessed based on the considered free stream velocity vector.

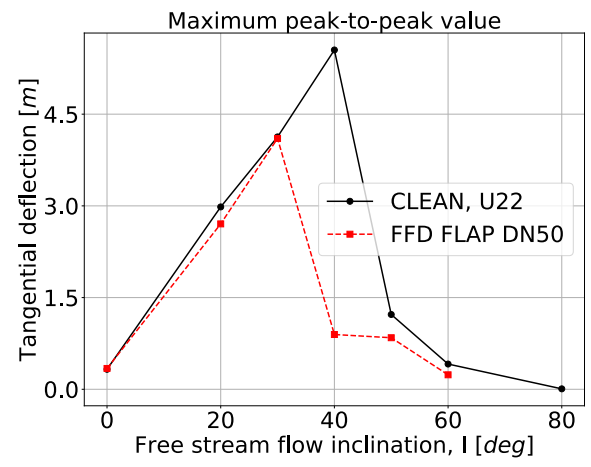

(a) Effect of inclination angle

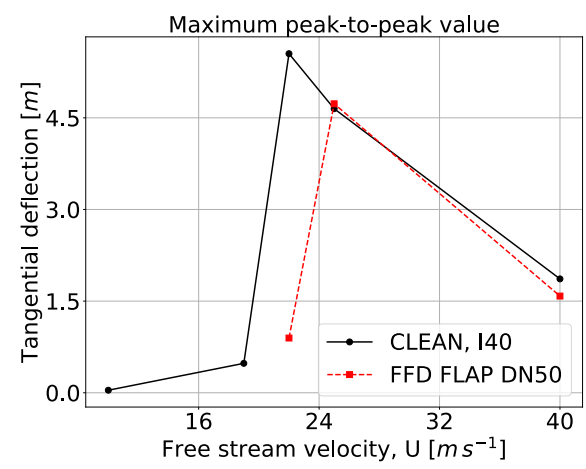

(b) Effect of free stream velocity

Figure 13: Maximum peak-to-peak value of the tangential blade tip deflection transients. Comparison of clean geometry and FFD flap working at $-50 \mathrm{deg}$.

\section{CONCLUSION}

This paper presents a computational study aiming to assess the performance of trailing edge flaps with regards to the suppression of Vortex Induced Vibrations (VIV) of large wind turbine blades. In particular the blade of the the AVATAR reference rotor was studied by means of 
a Fluid Structure Interaction (FSI) approach, coupling an Improved Delayed Detached Eddy Simulation (IDDES) solver with a beam model of the structure.

A first set of computations based on the clean blade geometry allowed to determine several inflow configurations triggering the VIV phenomenon. As for previous work included in the literature, two main parameters leading to the amplification of the blade edgewise vibrations were identified. On one hand, the chosen absolute value of the free stream velocity should be tuned in order to lead to natural shedding frequencies at the outboard region close to the first edgewise mode of the blade. As shown in this paper, a good initial estimation can be made by assuming a typical Strouhal number of 0.16. The second parameter required for the triggering of VIV was found to be the inclination angle of the flow with respect to the blade axis. Indeed, a significant spanwise correlation of the shedding frequencies of the different blade sections was achieved by considering inclination angles at the vicinity of $40 \mathrm{deg}$.

A second set of simulations accounted for trailing edge flaps, defined via an analytical expression of their geometry. The inflow conditions exhibiting the maximum amplification for the clean geometry were assumed. The performance of the flaps for VIV suppression was assessed, together with the influence of the geometric parameters characterizing this device. Several flap geometries did manage to suppress the blade vibrations amplification. Of particular interest was the operating angle $-30 \mathrm{deg}$, that resulted in very low blade tip deflection amplitudes regardless of the considered flap geometry. The mechanism preventing the VIV was concluded to be related to the suppression of the Von Karman street at the outboard region. Further studies would be required in order to completely understand this phenomenon. As a preliminary hypothesis, the change of the Strouhal number distribution in the outboard region induced by the flap geometry was argued.

The final set of simulations aimed to evaluate the performance of trailing edge flaps for VIV suppression in a wider range of inflow conditions. The initially studied analytical representation of the flap geometry was replaced by the use of a Free Form Deformation (FFD), allowing a much more versatile approach for future applications. A single flap operating angle, calibrated in previous simulations, was also used for all the new inflow conditions. The obtained results showed that, outside of the initially studied inflow conditions, the behaviour of the flapped geometry was equivalent to its clean counterpart. It was therefore concluded that a re-calibration of the flap operating angle should be required in order to adapt it to the inflow conditions. In view of the parallelism between the observed Von Karman street suppression and previous experiences regarding straked cylinders, the performance of the studied flaps could also be improved by considering variable operating angles in spanwise.

This first evaluation of the use of flaps for VIV suppression reveals a good potential for these flow control devices. However, a better understanding of the involved fluid structure interaction mechanisms should be achieved. This would allow for an identification of the best operating angle for every inflow configuration, together with a more realistic design of the flap geometries. In particular, stiff simulations of the flapped geometries would be required in order to evaluate the change in the Von Karman shedding patterns. The introduction of the FFD approach in the proposed numerical approach also allows for an easier exploration of new flow control devices as a future work, such as the so-called split flaps or a combination of trailing edge flaps working at different operating angles. 
S.G. Horcas, M.H.A. Madsen, N.N. Sørensen and F. Zahle

\section{REFERENCES}

Barlas, T., Jost, E., Pirrung, G., Tsiantas, T., Riziotis, V., Navalkar, S. T., Lutz, T., and Van Wingerden, J. W. (2016). Benchmarking aerodynamic prediction of unsteady rotor aerodynamics of active flaps on wind turbine blades using ranging fidelity tools. Journal of Physics: Conference Series, 753(2).

Gioria, R. S., Korkischko, I., and Meneghini, J. R. (2011). Simulation of Flow Around a Circular Cylinder Fitted With Strakes. In 21st International Congress of Mechanical Engineering.

Heinz, J. C., Sørensen, N. N., Riziotis, V., Schwarz, M., Gomez-iradi, S., and Stettner, M. (2016a). Aerodynamics of Large Rotors. WP4. Deliverable 4.5. Technical report, ECN Wind Energy, Petten, The Netherlands.

Heinz, J. C., Sørensen, N. N., Zahle, F., and Skrypinski, W. (2016b). Vortex-induced vibrations on a modern wind turbine blade. Wind Energy, 19(11):2041-2051.

Hoang, M. C., Laneville, A., and Légeron, F. (2015). Experimental study on aerodynamic coefficients of yawed cylinders. Journal of Fluids and Structures, 54:597-611.

Jost, E., Fischer, A., Lutz, T., and Krämer, E. (2016). An investigation of unsteady 3D effects on trailing edge flaps. Journal of Physics: Conference Series, 753(2).

Larsen, T. J. and Hansen, A. M. (2015). HAWC2, the user's manual. Technical Report July, Ris $\varnothing$.

Lekou, D., Chortis, D., Chaviaropoulos, P., Munduate, X., Irisarri, A., Madsen, H, A., Yde, K., Thomsen, K., Stettner, M., Reijerkerk, M., Grasso, F., Savenije, R., Schepers, G., and Andersen, C. (2015). AVATAR Deliverable D1.2: Reference Blade Design. Technical report, ECN Wind Energy, Petten, The Netherlands.

Matsumoto, M., Yagi, T., Shigemura, Y., and Tsushima, D. (2001). Vortex-induced cable vibration of cablestayed bridges at high reduced wind velocity. Journal of Wind Engineering and Industrial Aerodynamics, 89(7-8):633-647.

Menter, F. R. (1994). Two-equation eddy-viscosity turbulence models for engineering applications. AIAA Journal, 32(8):1598-1605.

Menter, F. R. and Kuntz, M. (2004). Adaptation of Eddy-Viscosity Turbulence Models to Unsteady Separated Flow Behind Vehicles. In McCallen, R., Browand, F., and Ross, J., editors, The Aerodynamics of Heavy Vehicles: Trucks, Buses, and Trains, pages 339-352. Springer Berlin Heidelberg.

Michelsen, J. A. (1992). Basis3D - a Platform for Development of Multiblock PDE Solvers. Technical Report AFM 92-05, Technical University of Denmark, Department of Fluid Mechanics, Technical University of Denmark.

Michelsen, J. A. (1994). Block structured Multigrid solution of 2D and 3D elliptic PDE's. Technical Report AFM 94-06, Technical University of Denmark, Department of Fluid Mechanics, Technical University of Denmark.

Sederberg, T. W. and Parry, S. R. (1986). Free-form deformation of solid geometric models. ACM SIGGRAPH Computer Graphics, 20(4):151-160.

Sørensen, N. N. (1995). General Purpose Flow Solver Applied to Flow over Hills. Ris $\varnothing-R-827-($ EN), Ris $\varnothing$ National Laboratory, Roskilde, Denmark.

Welch, P. (1967). The use of the fast Fourier transform for the estimation of power spectra: A method based on time averaging over short, modified periodograms. IEEE Trans. Audio Electroacoust, 15:70-73.

Yeo, D. and Jones, N. P. (2010). Aerodynamic Effects of Strake Patterns on Flow around a Yawed Circular Cylinder. In The Fifth International Symposium on Computational Wind Engineering (CWE2010), Chapel Hill, North Carolina, USA.

Zhou, T., Razali, S. F., Hao, Z., and Cheng, L. (2011). On the study of vortex-induced vibration of a cylinder with helical strakes. Journal of Fluids and Structures, 27(7):903-917. 